\title{
IDENTIFICACIÓN MOLECULAR Y REGISTRO DE Aphelenchoides spp. EN CULTIVOS COMERCIALES DE Hydrangea EN ANTIOQUIA, COLOMBIA
}

\section{MOLECULAR IDENTIFICATION AND REPORT OF Aphelenchoides spp. IN COMMERCIAL CROPS OF Hydrangea IN ANTIOQUIA, COLOMBIA}

Isabel Luna Piña ${ }^{1}$, Ana M. Olave Velandia ${ }^{2}$, Elkin López Arismendy ${ }^{3}$, William Cardona Garzon ${ }^{4}$, Juan F. Alzate Restrepo ${ }^{5}$

${ }^{1}$ Ing. Agrónoma, Facultad de Agronomía. Universidad Católica de Oriente, Rionegro-Antioquia, Colombia, (Dhttps://orcid. org/0000-0003-1818-8286; ${ }^{2}$ Microbióloga y Bionanalista, M.Sc. en Biología. Centro Nacional de Secuenciación Genómica-CNSG. SIU, Grupo de Parasitología, Facultad de Medicina. Universidad de Antioquia, Medellín, Colombia, (iDhttps://orcid.org/0000-0002-8817-9214; ${ }^{3}$ Bacteriólogo, M.Sc. en Biotecnología. SAFER AGROBIOLÓGICOS SAS, (iDhttps://orcid. org/0000-0001-5818-7793; ${ }^{4}$ Ing. Agrónomo. SAFER AGROBIOLÓGICOS SAS, (DiDhtps://orcid.org/0000-0001-9610-4135; ${ }^{5}$ Bacteriólogo, M.Sc. Ph.D. Centro Nacional de Secuenciación Genómica-CNSG. SIU, Grupo de Parasitología, Facultad de Medicina. Universidad de Antioquia, Medellín, Colombia, e-mail: jfernando.alzate@udea.edu.co, (iDhttps://orcid.org/00000003-2578-4609. Dirección de correspondencia: Centro Nacional de Secuenciación Genómica -CNSG, Unive rsidad de Antioquia UdeA, Calle 70 No. 52-21, Medellín-Antioquia, Colombia.

Rev. U.D.C.A Act. \& Div. Cient. 21(1):377-384, Julio-Diciembre 2018

https://doi.org/10.31910/rudca.v21.n2.2018.994

Artículo de acceso abierto publicado por Revista U.D.C.A Actualidad \& Divulgación Científica

bajo una licencia Creative Commons CC BY-NC 4.0

\section{RESUMEN}

El cultivo comercial de hortensias para flor de exportación ocupa un renglón importante en el sector económico del oriente antioqueño, por ser fuente de empleo y de desarrollo en la zona. La hortensia (Hydrangea macrophylla) es afectada por numerosos organismos fitopatógenos, entre ellos, nematodos del género Aphelenchoides, los que ocasionan, en el follaje, lesiones necróticas angulares, malformación de flor, enanismo y un daño indirecto en la tasa fotosintética, demeritando los parámetros de calidad para exportación. El objetivo de este estudio fue identificar, molecularmente, las especies del nematodo Aphelenchoides asociadas al cultivo de hortensias de color, en los municipios de Medellín (Santa Elena), La Ceja y Rionegro, siendo este el primer reporte para Colombia, de las especies de este género. Para la ejecución del estudio, se realizaron 10 muestreos en cultivos comerciales, distribuidos entre los tres municipios mencionados. Los nematodos extraídos, se sometieron a pruebas basadas en el análisis de ADN, haciendo uso del marcador ribosomal 18S. Los análisis filogenéticos practicados mostraron la presencia de la especie Aphelenchoides ritzemabosi en cultivos de hortensias, del corregimiento de Santa Elena y, de A. fragarie, en los municipios de La Ceja y Rionegro.
Palabras clave: Nematodo, fitopatógeno, hortensia, gen ribosomal $18 \mathrm{~S}$.

\section{ABSTRACT}

Hydrangea macrophylla commercial crops have an important economic significance for the eastern region of the department of Antioquia, Colombia, due to its impact on employment and local development. This flowering plant can be attacked by several phytopathogens among them nematodes of the genus Aphelenchoides; which can produce necrotic lesions on the plant leaves as well decreased photosynthetic rate, dwarfism, and flower malformation. Additionally, this pathogen is considered a regulated pest and limits the international commercialization of the flower. The aim of this study was to molecularly identify the Aphelenchoides species associated with infected $H$. macrophylla plants in crops the municipalities of Medellín, La Ceja, and Rionegro. This is the first report of the species of this phytopathogen for Colombia. The sampling activities were performed in ten commercial crops locate at the three municipalities. The isolated nematodes were subject to DNA-based tests were the 18S rDNA gene was amplified, sequenced and analyzed using phylogenetic methods. The obtained results showed 
the infection of Aphelenchoides ritzemabosi within the Santa Elena (Medellin) area in crops and A. fragarie within the municipalities of La Ceja, and Rionegro.

Keywords: Nematode, phytopathogen, Hydrangea, 18S rDNA.

\section{INTRODUCCIÓN}

El género Aphelenchoides fue descrito por Fisher en 1894 y, a la fecha, se han aceptado 180 especies; de estás, 13 han sido reportadas como parásitas de plantas y 19 aún están en estado incierto de clasificación (Sánchez-Monge et al. 2015; Golhasan et al. 2017). La mayoría de las especies de Aphelenchoides son fungívoras y asociadas a tejidos vegetales en descomposición; sin embargo, la discriminación de especies basado solo en características morfológicas es compleja (Yeates, 1999). Dentro de este género, se encuentran los comúnmente llamados nematodos foliares, causales de deformaciones y de lesiones necróticas irregulares en las hojas, con tres especies de reconocida importancia en plantas ornamentales y de cultivo: i) Aphelenchoides ritzemabosi (Schwartz, 1911): se ha descrito que parasita las hojas, brotes y el punto de crecimiento en plantas ornamentales, como el crisantemo; alimentándose de forma endoparásita del mesófilo. Este nematodo también se puede comportar como ectoparásito, afectando brotes y puntos de crecimiento (EPPO, 2017); ii) A. besseyi (Christie, 1942): causante del enanismo y rizado en fresa y de la enfermedad de las puntas blancas del arroz, que va acompañada de placas cloróticas, en la parte media de las hojas (Escuer et al. 2000; De Jesus et al. 2016); iii) A. fragariae (Ritzema Bos, 1890), Christie (1932), lo reporta como un parásito de las partes aéreas de las plantas, donde se puede comportar como endo y ectoparásito. Se ha descrito que puede afectar a más de 250 especies de plantas y también se puede encontrar asociado a plantas en bulbo o suelo. En las partes aéreas ocasiona clorosis o lesiones necróticas, delimitadas por las venas y, al final, defoliación. Es conocida vulgarmente como "nematodo del enanismo primaveral" o "nematodo de los helechos" (Escuer et al. 2000; Sánchez-Monge et al. 2015).

Otra especie de interés fitosanitario es A. subtenuis (Cobb, 1926), conocida como nematodo de bulbos y hojas, es una causa importante de enfermedad en narcisos (Narcissus) (Mor et al. 1993).

La hortensia Hydrangea macrophylla es originaria del Himalaya, China y Japón, a partir, de la cual, se han obtenido diferentes variedades de colores, para satisfacer los mercados de Estados Unidos y de Asia (Guérin, 2002; Weakley, 2015).

En el Oriente antioqueño, hace veinte años, que se sembró la primera hectárea de hortensia, llegando al 2014, a más de 1.000ha y ocupando el segundo puesto de los productos de exportación de la región (Rojas, 2014).

Precisamente, con el incremento de predios dedicados a la producción comercial de la hortensia en el Oriente antioqueño, se ha venido observando aumentos en las problemáticas fitosanitarias, entre ellas, una centrada en el área foliar; lesiones necróticas limitadas por la nervadura, acompañadas de entrenudos cortos y malformación de la flor, que comprometen el rendimiento y la rentabilidad del productor. Como agente asociado a esta sintomatología, el laboratorio de diagnósticos de la Universidad Católica de Oriente, en el 2015, aisló nematodos que, por morfología, fueron clasificados en el género Aphelenchoides (comunicación personal de datos no publicados).

La identificación de especies del género Aphelenchoides, se realiza a partir de la observación de características morfológicas de los nematodos adultos, que incluye la presencia de un metacorpus esférico, redondo-rectangular, muy grande, ocupando la mayor parte de la cavidad del cuerpo, tamaño entre 0,2 a $1,3 \mathrm{~mm}$ de longitud, cutícula ligeramente anulada, una glándula esofageal, usualmente superpuesta dorsalmente, estilete delgado, con perillas pequeñas, campo lateral con dos a cuatro líneas, vulva usualmente al 60-75\% del cuerpo, machos con espícula bien desarrollada, bursa ausente, posición de descanso de la hembra ligeramente arqueada, macho en forma de bastón en la región de la cola (Escuer et al. 2000; IPPC, 2016); sin embargo, estos parámetros requieren ser complementados con morfometría y microscopia electrónica y personal altamente especializado, para poder llevar la identificación, a nivel de especie (Shurtleff et al. 2000).

En este estudio nos propusimos identificar, con técnicas moleculares, las especies del nematodo Aphelenchoides, asociadas a cultivos comerciales de hortensia de color, en los municipios de Medellín, La Ceja y Rionegro (Antioquia), como una alternativa más precisa y menos compleja para la clasificación de especies para este género. Para dar cumplimiento a este objetivo, la comunidad internacional ha propuesto el uso de marcadores moleculares, como el gen ribosomal 18S. Este marcador ha mostrado ser lo suficientemente variable como para discriminar, entre las especies de importancia fitosanitaria y cuarentenaria (Bhadury et al. 2006; Rybarczyk-Mydlowska et al. 2012; Sánchez-Monge et al. 2017; Buonicontro et al. 2018).

La información obtenida con la identificación, a nivel de especie por métodos moleculares del nematodo Aphelenchoides, permitiría conocer, con certeza, la clase trófica del nematodo y su asociación o no a una patología que, puesta en manos de los técnicos y agricultores, les permitiría desarrollar planes de manejo. Además, esta es una herramienta muy 
útil, que permite identificar las especies consideradas como cuarentenarias, puesto que la producción de hortensia es en su totalidad para exportación y, actualmente, se desconocen las especies presentes en el Oriente antioqueño.

\section{MATERIALES Y MÉTODOS}

Selección de unidad experimental. El estudio, se realizó en tres municipios del Oriente antioqueño: Medellín (corregimiento de Santa Elena), La Ceja del Tambo y Rionegro, para un total de 10 muestreos, distribuidos en dichos municipios, en cultivos comerciales de 3 a 4 has de hortensia para exportación, variedad de color y en etapa de producción. En la tabla 1, se describen los sitios de muestreo, con los respectivos datos de georreferenciación.
Toma de muestras. Se hizo un recorrido aleatorio de lotes comerciales de hortensia de variedad de color - purple y azul-, con recolección de muestras sintomáticas, aproximadamente, $300 \mathrm{~g}$ de hojas, transportadas en bolsas de papel hasta el laboratorio, con su respectivo código (Tabla 1).

Procesamiento de extracción del nematodo. Los análisis de población y de caracterización de los nematodos fueron realizados en el laboratorio de Safer Agrobiológicos y Centro Nacional de Secuenciación Genómica-CNSG, Sede de Investigación Universitaria-SIU (Universidad de Antioquia). Para la extracción de nematodos foliares, a partir de material vegetal con alta infestación, se cortó el tejido en trozos pequeños y se dejó en remojo en agua, por una hora.

Tabla 1. Código y procedencia de las muestras que fueron ingresadas en el laboratorio, para la extracción del nematodo.

\begin{tabular}{|c|c|c|}
\hline Código & Procedencia del Material & Georeferenciación \\
\hline SF001 & Medellín, corregimiento Santa Elena & $6^{\circ} 12^{\prime} 50.9^{\prime \prime} \mathrm{N} 75^{\circ} 30^{\prime} 25.1^{\prime \prime} \mathrm{W}$ \\
\hline SF002 & Rionegro, vereda Tablazo & $6^{\circ} 07^{\prime} 32.6^{\prime \prime} \mathrm{N} 75^{\circ} 26^{\prime} 54.5^{\prime \prime} \mathrm{W}$ \\
\hline SF003 & Rionegro, vereda Tablazo & $6^{\circ} 07^{\prime} 32.6^{\prime \prime} \mathrm{N} 75^{\circ} 26^{\prime} 54.5^{\prime \prime} \mathrm{W}$ \\
\hline SF004 & Rionegro, vereda Tablazo & $6^{\circ} 07^{\prime} 32.6^{\prime \prime} \mathrm{N} 75^{\circ} 26^{\prime} 54.5^{\prime \prime} \mathrm{W}$ \\
\hline SF005 & La Ceja, vereda El Yarumo & $6^{\circ} 01^{\prime} 0.9^{\prime \prime} \mathrm{N} 75^{\circ} 23^{\prime 2} 23.9^{\prime \prime} \mathrm{W}$ \\
\hline SF006 & La Ceja, vereda El Yarumo & $6^{\circ} 01^{\prime} 0.9^{\prime \prime} \mathrm{N} 75^{\circ} 23^{\prime 2} 23.9^{\prime \prime} \mathrm{W}$ \\
\hline SF007 & La Ceja, vereda El Yarumo & $6^{\circ} 01^{\prime} 0.9^{\prime \prime} \mathrm{N} 75^{\circ} 23^{\prime 2} 23.9^{\prime \prime} \mathrm{W}$ \\
\hline SF012 & La Ceja, vereda El Tabor & $5^{\circ} 59^{\prime} 53.5^{\prime \prime} \mathrm{N} 75^{\circ} 24^{\prime} 54.5 ” \mathrm{~W}$ \\
\hline SF013 & Medellín, corregimiento Santa Elena & $6^{\circ} 12^{\prime} 50.9^{\prime \prime} \mathrm{N} 75^{\circ} 30^{\prime} 25.1 ” \mathrm{~W}$ \\
\hline SF014 & Rionegro, corregimiento Llanogrande & $6^{\circ} 06^{\prime} 35.0^{\prime \prime} \mathrm{N} 75^{\circ} 23^{\prime} 24.9^{\prime \prime} \mathrm{W}$ \\
\hline
\end{tabular}

Identificación morfológica. Los individuos encontrados se identificaron, siguiendo la clave descrita en el DP-17, por el IPPC International Plant Protection Convention (2016) y por Escuer et al. (2000), que refieren la presencia de bulbo medio esférico o redondo-rectangular, que ocupa casi toda la cavidad del cuerpo; región labial redondeada y ligeramente saliente del contorno del cuerpo; estilete delgado, pequeño, con nódulos poco desarrollados; vulva posterior, usualmente entre $60-75 \%$ de la longitud del cuerpo, presencia de mucro, como caracteres distintivos del género Aphelenchoides.

\section{Identificación molecular.}

Extracción del ADN. La extracción, se realizó usando el kit de Power Plant Pro DNA Isolation. Al finalizar el proceso de extracción, se hizo la cuantificación del ADN, por el método de absorción de luz, a 260nm (Nanodrop) y por picogreen. El ADN obtenido fue almacenado a $-20^{\circ} \mathrm{C}$.
Amplificación de un fragmento parcial del gen 18S. Para estos análisis, se utilizó, como marcador, el gen 18S, con los primers nem 1 5' GCAAGTCTGGTGCCAGCAGC 3' y nem2 5' CCGTGTTGAGTCAAATTAAG 3', los cuales, amplifican un fragmento entre 600 y 750 bp, aproximadamente, según la especie del nematodo.

Secuenciación. Cada uno de los amplicones obtenidos fue purificado y secuenciado por el método capilar/sanger, por ambas cadenas, con los mismos oligos nem1 y nem2. Los cromatogramas obtenidos fueron procesados con un umbral mínimo de calidad Q40 y se ensambló un contig, que fuera soportado por las regiones de mejor calidad, en ambas reacciones de secuenciamiento.

Análisis de clasificación. Con las secuencias obtenidas del gen 18S, se realizó una comparación con la base de datos $\mathrm{nr} / \mathrm{nt}$ del NCBI, usando el programa BLASTN, con el fin de 
confirmar que se amplificó el locus del gen ribosomal $18 \mathrm{~S}$. Posteriormente, se llevó a cabo un análisis filogenético complementario, que permitiera definir a qué grupo taxonómico pertenece cada una de estas secuencias, usando el programa MEGA versión 7.0 y un set de secuencias curadas de Aphelenchoides, de las especies descritas a la fecha (Tabla 2). Las secuencias, se alinearon con el programa MUSCLE, se calculó una matriz de distancias, se construyó un árbol filogenético, utilizando el método de Neighbor joining, con el modelo descrito por Kimura2, con distribución gama y con 1.000 réplicas de "bootstrap".

\section{RESULTADOS Y DISCUSIÓN}

Descripción de la patología observada y del nematodo encontrado. En los 10 muestreos realizados, se encontraron plantas de hortensia de la variedad purple, con síntomas de entrenudos cortos, formando casi una roseta, botón floral pequeño, con sépalos deformes, folíolos del tercio inferior y medio con lesiones lisas necróticas, definidas entre venas (lesiones angulares) (Figura 1) y con una severidad estimada del $50 \%$, del área foliar afectada.

Tabla 2. Código de acceso GenBank de las secuencias de Aphelenchoides, usadas como referencia en el análisis filogenético.

\begin{tabular}{|l|l|l|}
\hline \multicolumn{1}{|c|}{ Código acceso NCBI } & \multicolumn{1}{c|}{ Especie } & \multicolumn{1}{c|}{ País } \\
\hline JQ957877 & Aphelenchoides besseyi & Países bajos \\
\hline JQ957878 & Aphelenchoides besseyi & Países bajos \\
\hline AY284644 & Aphelenchoides blastophthorus & Países bajos \\
\hline JQ957879 & Aphelenchoides blastophthorus & Países bajos \\
\hline AB067755 & Aphelenchoides fragariae & Japón \\
\hline JQ957896 & Aphelenchoides fragariae & Países bajos \\
\hline EU325681 & Aphelenchoides fragariae & EUA \\
\hline DQ901551 & Aphelenchoides fragariae & EUA \\
\hline FJ520227 & Aphelenchoides fujianensis & China \\
\hline GU337993 & Aphelenchoides paradalianensis & China \\
\hline DQ901554 & Aphelenchoides ritzemabosi & EUA \\
\hline JQ957882 & Aphelenchoides ritzemabosi & Países bajos \\
\hline FJ040408 & Aphelenchoides saprophilus & Países bajos \\
\hline JQ957886 & Aphelenchoides subtenuis & Países bajos \\
\hline JQ957893 & Aphelenchoides subtenuis & Países bajos \\
\hline
\end{tabular}

Los nematodos asociados a la sintomatología fueron identificados por sus características morfológicas y morfométricas (Tabla 3), en el género Aphelenchoides; estos parámetros coinciden con los descritos por IPPC (2016), en el protocolo de diagnóstico DP-17.

Análisis de clasificación molecular. A partir de los nematodos adultos aislados, se logró purificar, exitosamente, ADN genómico, el cual, sirvió para amplificar el fragmento deseado del gen ribosomal 18S. En todos los casos, los primers funcionaron adecuadamente y se amplificó un fragmento de, aproximadamente, 600bp (Figura 2). El fragmento fue purificado y secuenciado por ambas cadenas, arrojando cromatogramas de calidad óptima, para los análisis subsiguientes.
Como un control preliminar, a las secuencias obtenidas, se realizó una comparación con las bases de datos $\mathrm{nr} / \mathrm{nt}$ del NCBI, usando el programa BLASTN, que mostró que las 10 muestras dieron hits cercanos, con secuencias del gen 18S, del género Aphelenchoides.

Para el análisis filogenético, se tomaron 25 secuencias de referencia del gen $18 \mathrm{~S}$ de miembros de la familia Aphelenchoididae, según se describe en la sección de métodos; se calculó una matriz de distancias filogenéticas y se construyó un árbol. De acuerdo con la matriz de distancia, las secuencias SF001 y SF0013 son más cercanas a Aphelenchoides ritzemabosi, con una distancia calculada de 0,000 . Las secuencias SF002, SF003, SF004, SF005, SF006, SF007, SF012 y SF014 son más cercanas a A. fragariae, con una distancia calculada de 0,000. 

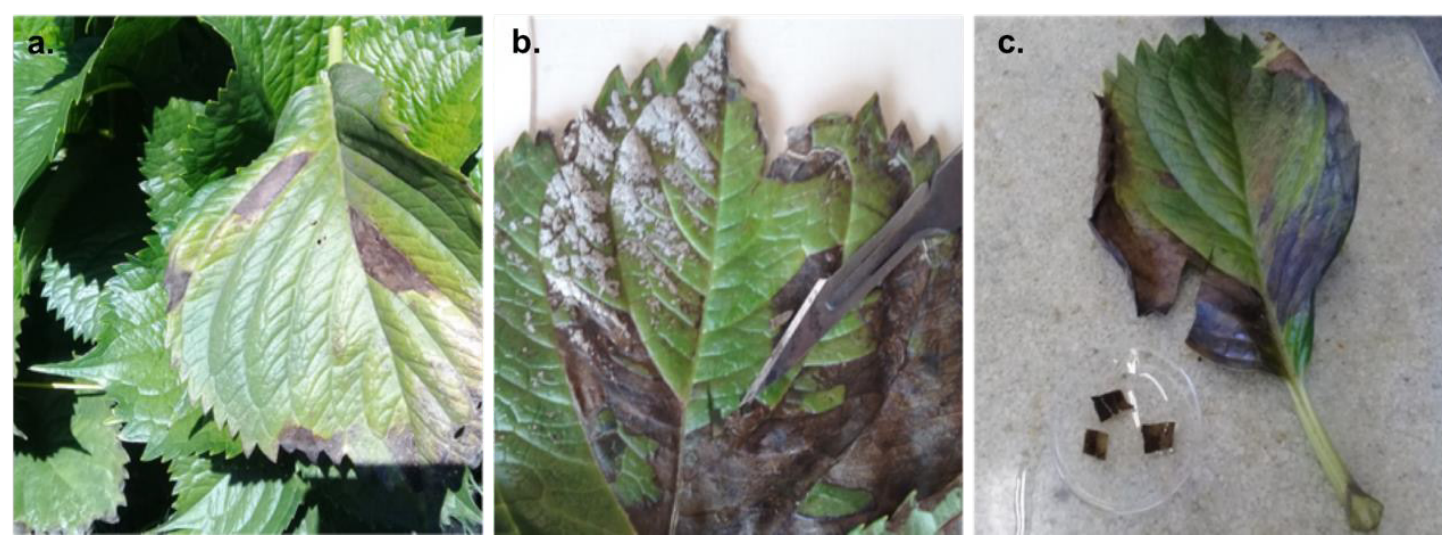

Figura 1. Fotografías que muestran las lesiones necróticas observadas en hojas de hortensias. a) Hoja infectada en su cultivo natural; b) Folíolos del tercio inferior y medio con lesiones lisas necróticas, definidas entre venas (lesiones angulares), con una severidad del 50\%, del área foliar afectada; c) Corte del tejido en trozos pequeños para la extracción de nematodos foliares, a partir de material vegetal con alta infestación.

Tabla 3. Características morfológicas de los nematodos aislados, según su procedencia.

\begin{tabular}{|l|l|l|}
\hline \multicolumn{1}{|c|}{ Característica } & Municipio de Medellín (Santa Elena) & Municipios de OA (La Ceja, Rionegro) \\
\hline Longitud del cuerpo & $0,73-0,93 \mathrm{~mm}$ & $0,68-0,7 \mathrm{~mm}$ \\
\hline Longitud del estilete & $0,015 \mathrm{~mm}$ & $0,046 \mathrm{~mm}$ \\
\hline Metacorpus & Largo y grande & Largo y grande \\
\hline Forma de la cola & Elongada conoide & Elongada conoide \\
\hline Mucro & Presente & Presente \\
\hline
\end{tabular}

OA: Oriente Antioqueño

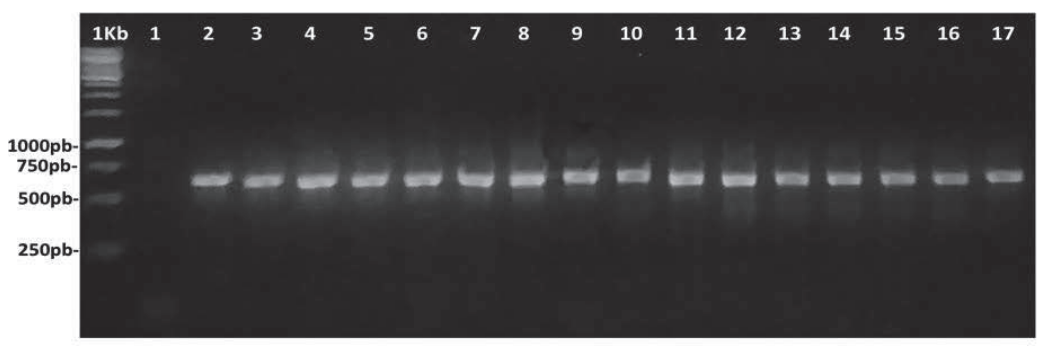

1. Control negativo PCR 10. SF001 60C

2. SF001 53ㄷ 11. SF002 $60 \% \mathrm{C}$

3. SF002 53 으 12. SF003 60 으

4. SF003 $53 \circ \mathrm{C} \quad$ 13. SF004 $60 \circ \mathrm{C}$

5. SF004 53ㅇ 14. SF005 $60 \% \mathrm{C}$

6. SF005 $53 \circ \mathrm{C} \quad$ 15. SF006 $60 \circ \mathrm{C}$

7. SF006 $53 \circ \mathrm{C}$ 16. SF007 $60 \circ \mathrm{C}$

8. SF007 $53 \circ \mathrm{C} \quad$ 17. SF008 $60 \circ \mathrm{C}$

9. $\mathrm{SF008} 53^{\circ} \mathrm{\circ} \mathrm{C}$

Figura 2. Electroforesis en gel de agarosa, para observar la amplificación por PCR, del gen rDNA 18S. El carril 1 es del control negativo de la PCR. Los carriles del 2 al 17 muestran preparaciones obtenidas con distintas temperaturas de annealing. Del carril 2 al 9, temperatura de alineamiento de primers, de $53^{\circ} \mathrm{C}$ y del carril 10 al 17 , de $60^{\circ} \mathrm{C}$. Se muestran resultados de 8 de las 10 muestras procesadas, con la respectiva amplificación de un fragmento, cercano a las 700 bp. 
El árbol construido dio lugar a dos subclados principales, con buenos soportes de bootstrap (Figura 3). En una clada, se encontraron A. ritzemabosi, A. besseyi, A. paradalian y A. fujianensis. En la otra clada principal, se encontraron las especies A. fragarie. A. blastophorus y A. saprophilus. Los aislados del corregimiento de Santa Elena y de los municipios de La Ceja y Rionegro, se asignaron con valores de soporte de bootstrap de 100 , a las especies A. ritzemabosi, en el caso de los aislados en Santa Elena y, A. fragarie, para La Ceja y Rionegro. Esto coincide con los resultados obtenidos en la matriz de distancias.
Las muestras SF002, SF003, SF004, SF005, SF006, SF007, SF0012 y SF0014 fueron confirmadas como aislados de la especie A. fragariae y las muestras SF001 y SF0013 fueron confirmadas como aislados de la especie A. ritzemabosi ( $\mathrm{Fi}-$ gura 3).

En el caso de Aphelenchoides, se ha publicado la capacidad del marcador ribosomal para resolver, como grupos monofiléticos, las especies descritas hasta el momento: A. ritzemabosi, A. fragariae, A. besseyi, A. fujianensis, A. blastophorus, A. subtenuis y A. saprophilus (De Jesus et al. 2016; Rybarczyk-Mydlowska et al. 2012).

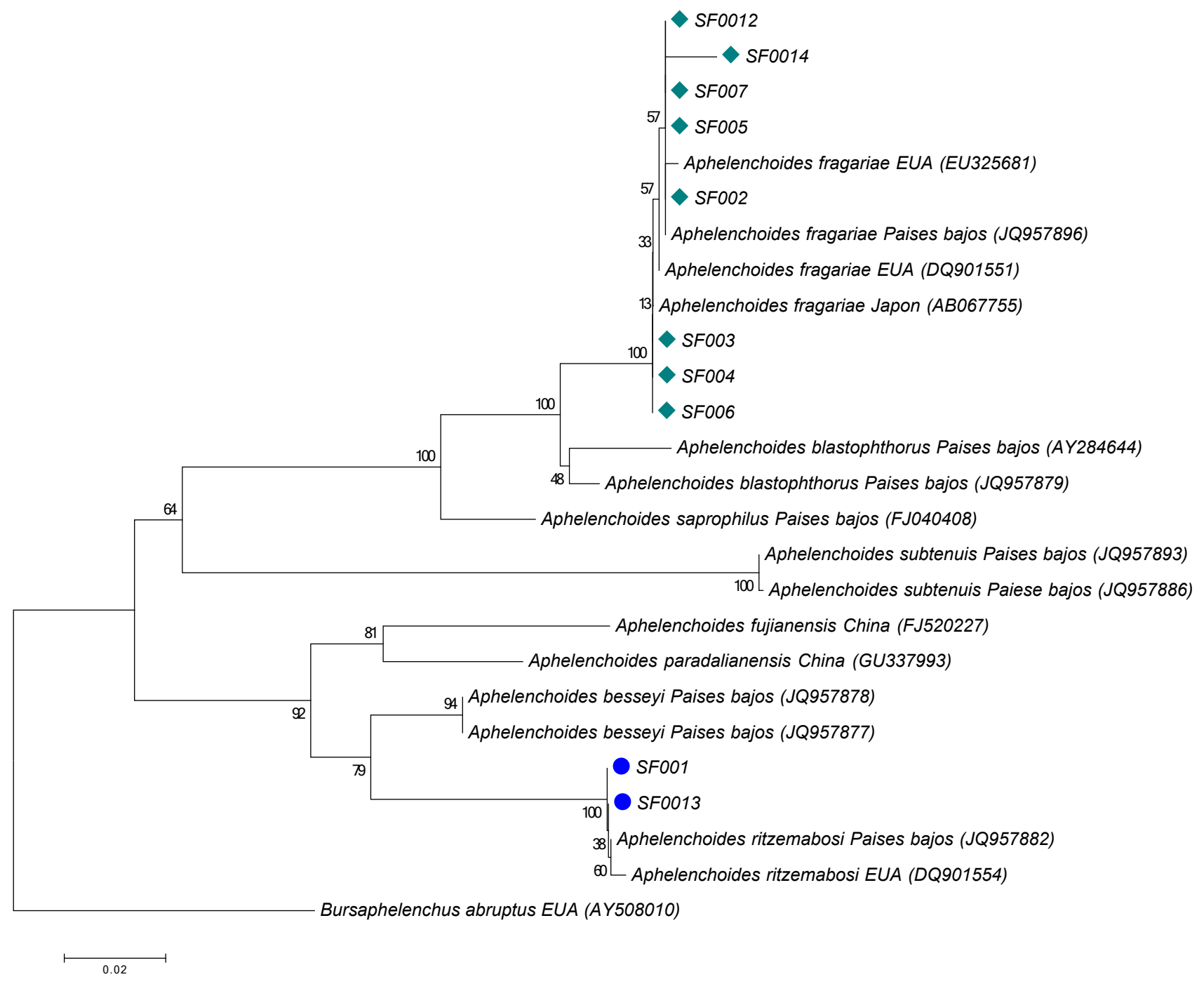

Figura 3. Árbol filogenético usando el marcador 18S rDNA. Las muestras de este estudio están marcadas con el código SFXXXX. Los círculos corresponden a los aislados clasificados como A. ritzemabosi y, los rombos, a A. fragariae. 
En el 2015, Sánchez-Monge et al. realizaron árboles filogenéticos, basados en tres marcadores moleculares, los cuales, se encontraban en gran parte de acuerdo entre sí, validando su uso para diagnóstico de Aphelenchoides. Adicionalmente, localizaron varias secuencias mal identificadas de Aphelenchoides, en bases de datos existentes. Estos autores concluyeron, que el análisis concatenado de los tres marcadores dio como resultado una visión más robusta de la filogenia y la evolución de Aphelenchoides, revelando que el parasitismo vegetal ha evolucionado de forma independiente, al menos tres veces, dentro de este género, presumiblemente, a partir de ancestros que se alimentan de hongos (Sánchez-Monge et al. 2015).

El diagnóstico integral, empleando técnicas morfológicas, morfométricas y moleculares, permiten un mejor estudio de las especies parásitas. En este estudio, aplicando técnicas moleculares, se logró hacer el primer reporte de $A$. fragariae y A. ritzemabosi, infectando plantas de hortensia en Colombia que, además de aportar al conocimiento de la Nematología del país, es un aporte base para las organizaciones nacionales de protección fitosanitarias (ONPF), para establecer normas regulatorias y negociaciones internacionales, para el tema de exportación. Especialmente, es el caso de Estados Unidos y de Chile, principales mercados adquisitivos de este ornamental, que tienen dentro de sus plagas reglamentadas y cuarentenarias, especies de este nematodo. Para el caso del sector floricultor, el ICA (ONPF, para Colombia), reglamentó la producción, la distribución y la comercialización de ornamentales, mediante la resolución 0264 de febrero de 2000 y estableció el mecanismo de registro de cultivos. Además, emitió una serie de decretos para el manejo de plagas de índole cuarentenario, según negociaciones realizadas con los mercados internacionales. A través de dicho mecanismo, se estableció el sistema de vigilancia fitosanitaria en cultivos ornamentales, que se actualiza constantemente, de acuerdo a las situaciones fitosanitarias del país.

La identificación correcta de las especies de Aphelenchoides asociadas a cultivos en nuestro país es esencial, para conocer su distribución y propagación e implementar estrategias de manejo. Asimismo, con la apertura de mercados internacionales para el comercio de cultivos ornamentales, existen implicaciones que pueden favorecer la introducción y la diseminación de especies de Aphelenchoides en los cultivos del país, no solo de tipo ornamental sino también en frutales, arroz, fresa y el fríjol. El primero de estos, con un reporte científico realizado por Hoyos-Carvajal et al. (2010), con base en claves taxonómicos hasta género.

El cultivo de hortensia, según registros del ICA, para el 2010, presentó un aumento significativo de intercepciones, por presencia de plagas (370). Esto se explica, en parte, dada la dinámica de las plagas, al movimiento comercial de ma- terial vegetal y porque la explotación comercial del cultivo se hace a campo abierto. Adicional a esto, el cultivo ha venido creciendo de forma acelerada, alcanzando más de 1.000 hectáreas sembradas en la región del Oriente antioqueño y siendo el $25 \%$ de la economía del municipio de la Ceja, según datos no oficiales de la alcaldía del municipio. Se desconoce la extensión del problema de Aphelenchoides en el cultivo de hortensias en Colombia, por lo que es necesario realizar prospecciones fitonematológicas en otras áreas productoras y otras variedades cultivadas. De esta manera, se podrá determinar la magnitud del daño y si las especies de Aphelenchoides asociadas son las mismas en todas las zonas. Finalmente, es necesario estar alerta a la infección de estos nematodos, sobretodo en época lluviosa, para evitar su diseminación en otros sitios donde se cultivan las hortensias, ya que, como afirman Cháves-Barrantes et al. (2014), el salpique de lluvia y el contacto entre plantas húmedas son fuente de diseminación del nematodo A. besseyi.

Conflictos de intereses: El manuscrito fue preparado y revisado con la participación de todos los autores, quienes declaramos que no existe conflicto de intereses que ponga en riesgo la validez de los resultados presentados. Financiación: Este estudio fue financiado con recursos de la vicerrectoría de investigación de la Universidad de Antioquia y por la empresa SAFER AGROBIOLÓGICOS SAS.

\section{REFERENCIAS}

1. BHADURY, P.; AUSTEN, M.; BILTON, D.; LAMBSHEAD, P.; ROGERS, A.; SMERDON, G. 2006. Development and evaluation of a DNA - barcoding approach for the rapid identification of nematodes. Inter Research Marine Ecology Progress series. 320:1-9. https://doi. org/10.3354/meps320001

2. BUIONICONTRO, D.; ROBERTS, D.; OLIVEIRA, C.; BLOK, V.; NEILSON, R.; OLIVEIRA, R. 2018. A rapid diagnostic for detection of Aphelenchoides besseyi and A. fujianensis based on real-time PCR. Plant Disease, 102(3):519-526. https://doi.org/10.1094/ PDIS-08-17-1160-RE

3. CHAVES-BARRANTES, N.; ARAYA-FERNÁNDEZ, C. 2014. Distribución espacial del amachamiento del frijol (Aphelenchoides besseyi Christie) en campo. Agronomía Mesoamericana. 25(1):13-21. https:// doi.org/10.15517/am.v25i1.14186

4. DE JESUS, D.; OLIVEIRA, C.; BALBINO, H.; MACKENZIE, K.; NEILSON, R.; PRIOR, T.; OLIVEIRA, R. 2016. Morphological and molecular characterisation of Aphelenchoides besseyi and A. fujianensis (Nematoda: Aphelenchoididae) from rice and forage 
grass seeds in Brazil. Nematology. 18(3):337-356. https://doi.org/10.1163/15685411-00002962

5. EPPO. 2017. PM 3/83 (1) Fragaria plants for planting inspection of places of production. Bulletin OEPP/ EPPO. Bulletin. 43:471-495.

6. ESCUER, M.; BELLO, A. 2000. Nematodos del género Aphelenchoides de interés fitopatológico y su distribución en España. Bol. San. Veg. Plagas. 26:47-63.

7. GOLHASAN, B.; HEYDARI, R.; ESMAEILI, M.; MIRAEIZ, E. 2017. Description of Aphelenchoides macrospica n. sp. (Nematoda: Aphelenchoididae) from Northwestern Iran. J. Nematology. 49(1):67-76. http:// doi.org/10.21307/jofnem-2017-046

8. GUÉRIN, V. 2002. Hydrangea: acquisitions nouvelles et applications. Un Point sur. - INRA. Paris, FRA: INRA Editions, 133p. https://prodinra.inra.fr/record/61858

9. HOYOS-CARVAJAL, L.; MOYA, J. 2010. Nematodos asociados con cultivos de arroz en Huila y Tolima. Agronomía Colombiana. 28(3):577-579.

10. IPPC. 2016. Annex to ISPM 27 - Aphelenchoides besseyi, A. fragariae and A. ritzemabosi (2006-025). Disponible desde Internet en: https:/www.ippc.int/ en/publications/83447/ (con acceso el 1/09/2016).

11. MOR, M; SPIEGEL, Y. 1993. Infection of Narcissus roots by Aphelenchoides subtenuis. J. Nematology. 25:476-479.

12. ROJAS, G. 2014. Antioquia es un jardín que florece todo el año. El Mundo. Disponible desde Internet en: http:/www.elmundo.com/portal/resultados/ detalles/?idx=241227 (con acceso el 20/04/2016).

13. RYBARCZYK-MYDLOWSKA, K.; MOOYMAN, P.; VAN MEGEN, H.; VAN DEN ELSEN, S.; VERVOORT, M.;
VEENHUIZEN, P.; VAN DOORN, J.; DEES, R.; KARSSEN, G.; BAKKER, J.; HELDER, H. 2012. Small subunit ribosomal DNA-based phylogenetic analysis of foliar nematodes (Aphelenchoides spp.) and their quantitative detection in complex DNA backgrounds. Phytopathology. 102:1153-1160. https:// doi.org/10.1094/PHYTO-05-12-0114-R

14. SÁNCHEZ-MONGE, A.; FLORES, L.; SALAZAR, L.; HOCKLAND, S.; BERT, W. 2015. An updated list of the plants associated with plant-parasitic Aphelenchoides (Nematoda: Aphelenchoididae) and its implications for plant-parasitism within this genus. Zootaxa. 4013(2):207-224. http://dx.doi.org/10.11646/ zootaxa.4013.2.3

15. SÁNCHEZ-MONGE, A.; JANSSEN, T.; FANG, Y.; COUVREUR, M.; KARSSEN, G.; BERT, W. 2017. mtCOI successfully diagnoses the four main plant-parasitic Aphelenchoides species (Nematoda: Aphelenchoididae) and supports a multiple origin of plant-parasitism in this paraphyletic genus. European J. Plant Pathology. 148(4):853-866. https://doi.org/10.1007/ s10658-016-1141-1

16. SHURTLEFF, M.; AVERRE III, C. 2000. Diagnosing plant diseases caused by nematodes. APS Press. 189p.

17. WEAKLEY, A.S. 2015. Flora of the Southern and Mid-Atlantic States. Working draft of 21 May 2015. Univ. of North Carolina Herbarium (NCU), Chapel Hill. Disponible desde Internet en: http://www.herbarium.unc. edu/flora.htm (con acceso el 1/09/2016).

18. YEATES, G. 1999. Effects of plants on nematode community structure. Ann. Rev. Phytopathol. 37(1):127-149. https://doi.org/10.1146/annurev.phyto.37.1.127

Recibido: Abril 20 de 2018

Aceptado: Octubre 18 de 2018

\section{Cómo citar:}

Luna Piña, I.; Olave Velandia, A.M.; López Arismendy, E.; Cardona Garzón, W.; Alzate Restrepo, J.F. 2018. Identificación molecular y registro de Aphelenchoides spp. en cultivos comerciales de Hydrangea en Antioquia, Colombia. Rev. U.D.C.A Act. \& Div. Cient. 21(2):377-384. https://doi.org/10.31910/rudca.v21.n2.2018.994 